\title{
New Positive Realness Conditions for Uncertain Discrete Descriptor Systems: Analysis and Synthesis
}

\author{
Shengyuan Xu and James Lam, Senior Member, IEEE
}

\begin{abstract}
This paper deals with the problems of positive real (PR) analysis and PR control for uncertain discrete-time descriptor systems. The parameter uncertainties are assumed to be time-invariant norm bounded and appear in both the state and input matrices. A new necessary and sufficient condition for a discrete-time descriptor system to be regular, causal, stable and extended strictly PR (ESPR) is proposed in terms of a strict linear matrix inequality. Based on this, the concepts of strong robust admissibility with ESPR and strong robust admissibilizability with ESPR were introduced. Without any additional assumptions on the system matrices, necessary and sufficient conditions for strong robust admissibility with ESPR and strong robust admissibilizability with ESPR are obtained. Through these results, the problems of PR analysis and PR control are solved. Furthermore, an explicit expression of a desired state feedback controller is also given, which involves no decomposition of the system matrices.
\end{abstract}

Index Terms-Descriptor systems, discrete-time systems, parameter uncertainty, positive real (PR) control, state feedback.

\section{INTRODUCTION}

$\mathbf{P}$ OSITIVE real (PR) control is a research problem of recurring interest in the past years [1], [3], [10]. The study of this problem is motivated by the robust and nonlinear control where a well-known fact is that the positive realness of a certain loop transfer function will guarantee the overall stability of a feedback system if uncertainty or nonlinearity can be characterized by a PR system [12]. By solving a pair of Riccati inequalities, a solution to this problem was given in [10]. When parameter uncertainties appear in a system model, the PR control problem was studied in [14], where state feedback controllers were designed and a Riccati equation approach was developed, while in [7] observer-based dynamic output-feedback controllers were constructed in terms of solutions to certain linear matrix inequalities (LMIs). The corresponding results for discrete-time systems can be found in [8]. It is worth mentioning that the results in [8] were further extended to two-dimensional (2-D) systems in [16].

On the other hand, it is known that descriptor system models are more convenient and natural than state-space models in the

Manuscript received April 14, 2003; revised March 20, 2004. This work was supported by the Research Grants Council under Grant HKU 7103/01P, by the Foundation for the Author of National Excellent Doctoral Dissertation, China, under Grant 200240, by the National Natural Science Foundation, China, under Grant 60304001 and Grant 60074007, and by the Fok Ying Tung Education Foundation under Grant 91061 . This paper was recommended by Associate Editor R. Dogaru.

$\mathrm{S}$. Xu is with the Department of Automation, Nanjing University of Science and Technology, Nanjing 210094, China.

J. Lam is with the Department of Mechanical Engineering, University of Hong Kong, Hong Kong (e-mail: james.lam@hku.hk).

Digital Object Identifier 10.1109/TCSI.2004.834524 description of many practical systems, such as interconnected large-scale systems, economic systems, power systems [2], [5]. Descriptor systems are also referred to as singular systems, implicit systems, generalized state-space systems, differential-algebraic systems or semi-state systems. The study of descriptor systems has received much attention during the past decades, and many results based on the theory of state-space systems have been extended to the area of descriptor systems [2], [5], [6], [19]. Very recently, the positive realness of descriptor systems was studied in [21], where PR lemmas for descriptor systems in both the continuous and discrete contexts have been proposed in terms of generalized algebraic Riccati equations and inequalities. However, the design of controllers that achieve the positive realness of the closed-loop system has not been investigated in [21]. It is worth pointing out that the problem of PR control for discrete descriptor systems is much more difficult than that for state-space systems due to the fact that the Lyapunov-type matrix in discrete-time descriptor systems is indefinite as reported in [21] while in the state-space case the Lyapunov matrix is positive definite. It is also worth mentioning that in [21] parameter uncertainties in descriptor system model have not been considered.

In this paper, we consider the problems of PR analysis and PR control for uncertain discrete-time descriptor systems. The parameter uncertainties are assumed to be time invariant and unknown but norm bounded appearing in both the state and input matrices. In order to overcome the difficulty encountered when designing state feedback controllers for discrete-time descriptor systems, we first present a new necessary and sufficient condition in terms of a strict LMI, which ensures a discrete-time descriptor system to be regular, causal, stable and extended strictly $P R$ (ESPR). It should be pointed out that the given strict LMI is more desirable than a nonstrict one in [21] since testing a nonstrict LMI may cause some numerical problems [11]. In order to solve the problems, the concepts of strong robust admissibility with ESPR and strong robust admissibilizability with ESPR were introduced. Without assumptions on the system matrices, necessary and sufficient conditions for strong robust admissibility with ESPR and strong robust admissibilizability with ESPR are derived in terms of a strict LMI and matrix inequalities, respectively. When these matrix inequalities are feasible, a desired state feedback controller can be constructed directly and no decomposition of the system matrices is involved.

\section{A. Notation}

Throughout this paper, for real symmetric matrices $X$ and $Y$, the notation $X \geq Y$ (respectively, $X>Y$ ) means that the matrix $X-Y$ is positive semi-definite (respectively, positive def- 
inite). $I$ is the identity matrix with appropriate dimension. The superscripts " $T$ " and "*" represent the transpose and the complex conjugate transpose, respectively. The notation $D_{\text {int }}(0,1)$ is the interior of the unit disk with center at the origin. We use $\lambda(E, A)$ to represent the set $\{z \mid \operatorname{det}(z E-A)=0\}$. Matrices, if not explicitly stated, are assumed to have compatible dimensions.

\section{DEFINITIONS AND PROBLEM FORMULATION}

Consider an uncertain linear discrete-time descriptor system described by

$$
\begin{aligned}
(\Sigma): E x(k+1) & =(A+\Delta A) x(k)+\left(B_{1}+\Delta B_{1}\right) u(k)+B \omega(k) \\
z(k) & =C x(k)+D \omega(k)
\end{aligned}
$$

where $x(k) \in \mathbb{R}^{n}$ is the state; $u(k) \in \mathbb{R}^{m}$ is the control input; $\omega(k) \in \mathbb{R}^{l}$ is the disturbance input; and $z(k) \in \mathbb{R}^{q}$ is the controlled output. The matrix $E \in \mathbb{R}^{n \times n}$ may be singular; we assume that rank $E=r \leq n$, and $q=l . A, B, B_{1}, C$ and $D$ are known real constant matrices with appropriate dimensions. $\Delta A$ and $\Delta B$ are time-invariant matrices representing norm-bounded parameter uncertainties, and are assumed to be of the form

$$
\left[\begin{array}{ll}
\Delta A & \Delta B_{1}
\end{array}\right]=\operatorname{MF}(\sigma)\left[\begin{array}{ll}
N_{1} & N_{2}
\end{array}\right]
$$

where $M, N_{1}$, and $N_{2}$ are known real constant matrices with appropriate dimensions. The uncertain matrix $F(\sigma)$ satisfies

$$
F(\sigma) F(\sigma)^{T} \leq I
$$

and $\sigma \in \Xi$, where $\Xi$ is a compact set in $\mathbb{R}$. Furthermore, it is assumed that given any matrix $F: F F^{T} \leq I$, there exists a $\sigma \in \Xi$ such that $F=F(\sigma) . \Delta A$ and $\Delta B$ are said to be admissible if both (3) and (4) hold.

Remark 1: It should be pointed out that the structure of the uncertainty with the form (3) and (4) has been widely used when dealing with the problem of robust control for regular and descriptor uncertain systems in both continuous- and discrete-time contexts; see e.g., [13], [20], and the references therein.

The nominal discrete-time descriptor system of $(\Sigma)$ with $u(k)=0$ is

$$
\begin{aligned}
(\hat{\Sigma}): \quad E x(k+1) & =A x(k)+B \omega(k) \\
z(k) & =C x(k)+D \omega(k) .
\end{aligned}
$$

For the descriptor discrete-time system $(\hat{\Sigma})$, we introduce the following definition.

Definition 1-[2], [5]:

(I) $(\hat{\Sigma})$ is said to be regular if $\operatorname{det}(z E-A)$ is not identically zero.

(II) $(\hat{\Sigma})$ is said to be causal if the degree of $\operatorname{det}(z E-A)$ is equivalent to rank $E$.
(III) $(\hat{\Sigma})$ is said to be stable if $\lambda(E, A) \subset D_{\text {int }}(0,1)$.

(IV) $(\hat{\Sigma})$ is said to be admissible if it is regular, causal and stable.

When system $(\hat{\Sigma})$ is regular, the transfer function of this system is given as follows:

$$
G(z)=C(z E-A)^{-1} B+D
$$

Throughout this paper, we shall use the following concept of positive realness.

Definition 2-[21]:

(I) $\operatorname{System}(\hat{\Sigma})$ is said to be PR if its transfer function $G(z)$ is analytic in $|z|>1$ and satisfies $G(z)+G^{*}(z) \geq 0$ for $|z|>1$.

(II) System $(\hat{\Sigma})$ is said to be strictly PR (SPR) if its transfer function $G(z)$ is analytic in $|z| \geq 1$ and satisfies $G\left(e^{j \theta}\right)+G^{*}\left(e^{j \theta}\right)>0$ for $\theta \in[0,2 \pi)$.

(III) $\operatorname{System}(\hat{\Sigma})$ is said to be ESPR if it is SPR and $G(\infty)+$ $G(\infty)^{T}>0$.

The problems of PR analysis and PR control for the uncertain discrete-time descriptor system $(\Sigma)$ will be addressed. The purpose of the PR analysis problem is to develop conditions ensuring that the uncertain descriptor system $(\Sigma)$ with $u(k)=0$ is admissible and ESPR for all parameter uncertainty $\Delta A$ satisfying (3) and (4), while the aim of the PR control problem is to design a state feedback controller for $(\Sigma)$ such that the resulting closed-loop system is admissible and ESPR.

\section{MAIN RESULTS}

We first give the following results, which will be used in the derivation of our main results.

Lemma 1: The discrete-time descriptor system $(\hat{\Sigma})$ is admissible if and only if there exist matrices $P>0$ and $Q$ such that

$$
A^{T} P A-E^{T} P E+Q S^{T} A+A^{T} S Q^{T}<0
$$

where $S \in \mathbb{R}^{n \times(n-r)}$ is any matrix with full column and satisfies $E^{T} S=0$.

Proof: For the proof of Lemma 1, see [15].

Lemma 2-[17]: Given any matrices $X, Y$ and $Z$ with appropriate dimensions such that $Y>0$. Then, we have

$$
X^{T} Z+Z^{T} X+X^{T} Y X \geq-Z^{T} Y^{-1} Z
$$

Lemma 3-[4], [21]: Consider a state-space system described by

$$
\begin{aligned}
x(k+1) & =A x(k)+B \omega(k) \\
z(k) & =C x(k)+D \omega(k) .
\end{aligned}
$$

Then, this system is stable and ESPR if and only if there exists a matrix $P>0$ such that

$$
\left[\begin{array}{cc}
A^{T} P A-P & C^{T}-A^{T} P B \\
C-B^{T} P A & -\left(D^{T}+D-B^{T} P B\right)
\end{array}\right]<0 .
$$


Theorem 4: The discrete-time descriptor system $(\hat{\Sigma})$ is admissible and ESPR if and only if there exist matrices $P>0$ and $Q$ such that the following LMI holds:

$$
\begin{gathered}
{\left[\begin{array}{cc}
A^{T} P A-E^{T} P E & C^{T}-A^{T} P B \\
C-B^{T} P A & -\left(D^{T}+D-B^{T} P B\right)
\end{array}\right]} \\
+\left[\begin{array}{c}
A^{T} \\
-B^{T}
\end{array}\right] S Q^{T}+Q S^{T}\left[\begin{array}{c}
A^{T} \\
-B^{T}
\end{array}\right]^{T}<0
\end{gathered}
$$

where $S \in \mathbb{R}^{n \times(n-r)}$ is any matrix with full column and satisfies $E^{T} S=0$.

Proof: For the proof of Lemma 1, see the Appendix .

Remark 2: Theorem 1 provides a necessary and sufficient condition for the discrete-time descriptor system $(\hat{\Sigma})$ to be admissible and ESPR. In the case when $E=I$, that is, the descriptor system $(\hat{\Sigma})$ reduces to a state-space system, Theorem 1 coincides with Lemma 4.2 in [4]. Therefore, Theorem 1 can be regarded as an extension of existing ESPR results for discrete-time state-space systems to descriptor systems.

Remark 3: Note that the condition in (11) is a strict LMI, which is in contrast to those in [21], where nonstrict LMI conditions were given. It should be pointed out that some numerical problems may arise when checking nonstrict LMI conditions. Therefore, the strict LMI condition in (11) is more desirable from the numerical point of view.

Considering Theorem 1, we introduce the following definitions in order to solve the problems of PR analysis and PR control formulated in the previous section.

Definition 3: The uncertain discrete-time descriptor system $(\Sigma)$ with $u(k) \equiv 0$ is said to be strongly robustly admissible with ESPR if there exist matrices $P>0$ and $Q$ such that

$$
\begin{aligned}
& {\left[\begin{array}{cc}
(A+\Delta A)^{T} P(A+\Delta A)-E^{T} P E & C^{T}-(A+\Delta A)^{T} P B \\
C-B^{T} P(A+\Delta A) & -\left(D^{T}+D-B^{T} P B\right)
\end{array}\right]} \\
& +\left[\begin{array}{c}
(A+\Delta A)^{T} \\
-B^{T}
\end{array}\right] S Q^{T}+Q S^{T}\left[\begin{array}{c}
(A+\Delta A)^{T} \\
-B^{T}
\end{array}\right]^{T}<0
\end{aligned}
$$

for all the parameter uncertainty $\Delta A$ satisfying (3) and (4), where $S \in \mathbb{R}^{n \times(n-r)}$ is any matrix with full column and satisfies $E^{T} S=0$.

Definition 4: The uncertain discrete-time descriptor system $(\Sigma)$ is said to be strongly robustly admissibilizable with ESPR if there exists a linear state feedback control law $u(k)=K x(k)$, $K \in \mathbb{R}^{m \times n}$, such that the closed-loop system is strongly robustly admissible with ESPR in the sense of Definition 3.

In the following, attention will be focused on the development of conditions for strong robust admissibility with ESPR and strong robust admissibilizability with ESPR, respectively. To this end, we need the following result.

Lemma 4: [9] Given matrices $\Omega, \Gamma$ and $\Xi$ of appropriate dimensions and with $\Omega$ symmetric, then

$$
\Omega+\Gamma F(\sigma) \Xi+(\Gamma F(\sigma) \Xi)^{T}<0
$$

for all $F(\sigma)$ satisfying $F(\sigma) F(\sigma)^{T} \leq I$, if and only if there exists a scalar $\epsilon>0$ such that

$$
\Omega+\epsilon \Gamma \Gamma^{T}+\epsilon^{-1} \Xi^{T} \Xi<0 .
$$

Now, we are in a position to present a necessary and sufficient condition for strong robust admissibility with ESPR.

Theorem 2: The uncertain discrete-time descriptor system $(\Sigma)$ with $u(k)=0$ is strongly robustly admissible with ESPR if and only if there exist a scalar $\epsilon>0$, matrices $P>0$ and $Q$ such that the following LMI holds:

$$
\left[\begin{array}{ccc}
\Pi_{1}+\tilde{\Pi}_{1} & \Pi_{2} & \Pi_{3} M \\
\Pi_{2}^{T} & -P & P M \\
M^{T} \Pi_{3}^{T} & M^{T} P & -\epsilon I
\end{array}\right]<0
$$

where $\Pi_{1}, \Pi_{2}$, and $\Pi_{3}$ are given in (14)-(16), respectively, shown at the bottom of the page, and the matrix $S \in \mathbb{R}^{n \times(n-r)}$ is given in Definition 3.

Proof: By Definition 3, it is easy to see that the unforced discrete-time descriptor system of $(\Sigma)$ is strongly robustly admissible with ESPR if and only if there exist matrices $P>0$ and $Q$ such that (12) holds. Applying the Schur complement formula to (12), it can be seen that (12) is equivalent to (17) shown at the bottom of the page. Observe that (17) can be rewritten as

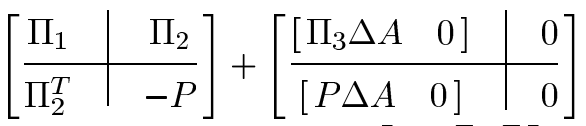

$$
\begin{aligned}
& +\left[\begin{array}{c|c}
{\left[\begin{array}{c}
\Delta A^{T} \Pi_{3}^{T} \\
0
\end{array}\right]} & {\left[\begin{array}{c}
\Delta A^{T} P \\
0
\end{array}\right]} \\
\hline 0 & 0
\end{array}\right]<0 .
\end{aligned}
$$

$$
\begin{aligned}
& \Pi_{1}=\left[\begin{array}{cc}
-E^{T} P E & C^{T}-A^{T} P B \\
C-B^{T} P A & -\left(D^{T}+D-B^{T} P B\right)
\end{array}\right]+\left[\begin{array}{c}
A^{T} \\
-B^{T}
\end{array}\right] S Q^{T}+Q S^{T}\left[\begin{array}{c}
A^{T} \\
-B^{T}
\end{array}\right]^{T} \\
& \tilde{\Pi}_{1}=\left[\begin{array}{cc}
\epsilon N_{1}^{T} N_{1} & 0 \\
0 & 0
\end{array}\right] \quad \Pi_{2}=\left[\begin{array}{c}
A^{T} P \\
0
\end{array}\right] \\
& \Pi_{3}=\left[\begin{array}{c}
0 \\
-B^{T} P
\end{array}\right]+Q S^{T}
\end{aligned}
$$

$$
\left[\begin{array}{cc}
\Pi_{1}+\left[\begin{array}{cc}
0 & -\Delta A^{T} P B \\
-B^{T} P \Delta A & 0
\end{array}\right]+\left[\begin{array}{c}
\Delta A^{T} S Q^{T} \\
0
\end{array}\right]+\left[\begin{array}{c}
\Delta A^{T} S Q^{T} \\
0
\end{array}\right]^{T} \mid\left[\begin{array}{c}
(A+\Delta A)^{T} P \\
0
\end{array}\right] \\
\hline[P(A+\Delta A) & 0]
\end{array}\right]<0 .
$$


That is

$$
\begin{aligned}
{\left[\begin{array}{cc}
\Pi_{1} & \Pi_{2} \\
\Pi_{2}^{T} & -P
\end{array}\right]+\left[\begin{array}{c}
\Pi_{3} M \\
P M
\end{array}\right] F(\sigma)\left[\begin{array}{ll}
\tilde{N} & 0
\end{array}\right] } \\
+\left(\left[\begin{array}{c}
\Pi_{3} M \\
P M
\end{array}\right] F(\sigma)\left[\begin{array}{ll}
\tilde{N} & 0
\end{array}\right]\right)^{T}<0
\end{aligned}
$$

where

$$
\tilde{N}=\left[\begin{array}{ll}
N_{1} & 0
\end{array}\right]
$$

By Lemma 4, it can be shown that (18) holds for all $F(\sigma)$ satisfying (4) if and only if there exists a scalar $\epsilon>0$ such that

$$
\left[\begin{array}{cc}
\Pi_{1} & \Pi_{2} \\
\Pi_{2}^{T} & -P
\end{array}\right]+\epsilon^{-1}\left[\begin{array}{c}
\Pi_{3} M \\
P M
\end{array}\right]\left[\begin{array}{c}
\Pi_{3} M \\
P M
\end{array}\right]^{T}+\epsilon\left[\begin{array}{c}
\tilde{N}^{T} \\
0
\end{array}\right]\left[\begin{array}{c}
\tilde{N}^{T} \\
0
\end{array}\right]^{T}<0
$$

which, by the Schur complement formula, results in the LMI in (13). This completes the proof.

The following theorem gives the result on the strong robust admissibilizability with ESPR.

Theorem 3: The uncertain discrete-time descriptor system $(\Sigma)$ is strongly robustly admissibilizable with ESPR if and only if there exist scalars $\epsilon>0, \delta>0$, matrices $P>0, Q_{1}$ and $Q_{2}$ such that

$$
\begin{aligned}
\mathcal{Z}= & P^{-1}-\epsilon^{-1} M M^{T}>0 \\
\mathcal{X}= & D^{T}+D-B^{T} P B+Q_{2} S^{T} B+B^{T} S Q_{2}^{T}-\epsilon^{-1}\left(Q_{2} S^{T}\right. \\
& \left.-B^{T} P\right) M\left(I+\epsilon^{-1} M^{T} \mathcal{Z}^{-1} M\right) M^{T}\left(S Q_{2}^{T}-P B\right)>0
\end{aligned}
$$

and

$\mathcal{Y}_{1}+\mathcal{Y}_{2} \mathcal{X}^{-1} \mathcal{Y}_{2}^{T}-\left(\Psi_{1}^{T}+\mathcal{Y}_{2} \mathcal{X}^{-1} \Psi_{2}^{T}\right) \Xi^{-1}\left(\Psi_{1}+\Psi_{2} \mathcal{X}^{-1} \mathcal{Y}_{2}^{T}\right)<0$ where the matrix $S \in \mathbb{R}^{n \times(n-r)}$ is given in Definition 3, and

$$
\begin{aligned}
\mathcal{Y}_{1}= & \epsilon N_{1}^{T} N_{1}+Q_{1} S^{T} A+A^{T} S Q_{1}^{T}-E^{T} P E \\
& +\epsilon^{-1} Q_{1} S^{T} M M^{T} S Q_{1}^{T} \\
& +\left(A^{T}+\epsilon^{-1} Q_{1} S^{T} M M^{T}\right) \mathcal{Z}^{-1}\left(A+\epsilon^{-1} M M^{T} S Q_{1}^{T}\right) \\
\mathcal{Y}_{2}= & C^{T}-Q_{1} S^{T} B+\left(A^{T}+\epsilon^{-1} Q_{1} S^{T} M M^{T}\right) \\
& \times\left(I+\epsilon^{-1} \mathcal{Z}^{-1} M M^{T}\right)\left(S Q_{2}^{T}-P B\right) \\
\Psi_{1}= & \epsilon N_{2}^{T} N_{1}+B_{1}^{T} \mathcal{Z}^{-1} A+B_{1}^{T}\left(I+\epsilon^{-1} \mathcal{Z}^{-1} M M^{T}\right) S Q_{1}^{T} \\
\Psi_{2}= & B_{1}^{T}\left(I+\epsilon^{-1} \mathcal{Z}^{-1} M M^{T}\right)\left(S Q_{2}^{T}-P B\right) \\
\Xi= & \epsilon N_{2}^{T} N_{2}+B_{1}^{T} \mathcal{Z}^{-1} B_{1}+\Psi_{2} \mathcal{X}^{-1} \Psi_{2}^{T}+\delta I .
\end{aligned}
$$

In this case, a state feedback control law chosen by

$$
u(k)=-\Xi^{-1}\left(\Psi_{1}+\Psi_{2} \mathcal{X}^{-1} \mathcal{Y}_{2}^{T}\right) x(k)
$$

will be such that the closed-loop system is strongly robustly admissible with ESPR.

Proof: By Definition 4 and Theorem 2, we have that the uncertain discrete-time descriptor system $(\Sigma)$ is strongly robustly admissibilizable with ESPR if and only if there exist a scalar $\epsilon>0$, matrices $P>0, Q$ and $K \in \mathbb{R}^{m \times n}$ such that

$$
\left[\begin{array}{ccc}
\hat{\Pi}_{1}+\tilde{\Pi}_{1} & \hat{\Pi}_{2} & \Pi_{3} M \\
\hat{\Pi}_{2}^{T} & -P & P M \\
M^{T} \Pi_{3}^{T} & M^{T} P & -\epsilon I
\end{array}\right]<0
$$

where $\Pi_{3}$ is given in (16), and $\Pi_{1} \hat{\Pi}_{1}, A_{c}$, and $N_{c}$ are given in (25)-(27) at the bottom of the page, respectively. Write

$$
Q=\left[\begin{array}{l}
Q_{1} \\
Q_{2}
\end{array}\right]
$$

where the partition is compatible with the related matrices. Then, (24) can be rewritten as the last equation at the bottom of the page, which, by the Schur complement formula again,

$$
\begin{aligned}
& \Pi_{1}=\left[\begin{array}{cc}
-E^{T} P E & C^{T}-A_{c}^{T} P B \\
C-B^{T} P A_{c} & -\left(D^{T}+D-B^{T} P B\right)
\end{array}\right]+\left[\begin{array}{c}
A_{c}^{T} \\
-B^{T}
\end{array}\right] S Q^{T}+Q S^{T}\left[\begin{array}{c}
A_{c}^{T} \\
-B^{T}
\end{array}\right]^{T} \\
& \tilde{\Pi}_{1}=\left[\begin{array}{cc}
\epsilon N_{c}^{T} N_{c} & 0 \\
0 & 0
\end{array}\right], \quad \hat{\Pi}_{2}=\left[\begin{array}{c}
A_{c}^{T} P \\
0
\end{array}\right] \\
& A_{c}=A+B_{1} K \quad N_{c}=N_{1}+N_{2} K
\end{aligned}
$$

$$
\left[\begin{array}{cccc}
\epsilon N_{c}^{T} N_{c}+Q_{1} S^{T} A_{c}+A_{c}^{T} S Q_{1}^{T}-E^{T} P E & C^{T}-A_{c}^{T}\left(P B-S Q_{2}^{T}\right)-Q_{1} S^{T} B & A_{c}^{T} P & Q_{1} S^{T} M \\
C-\left(B^{T} P-Q_{2} S^{T}\right) A_{c}-B^{T} S Q_{1}^{T} & -\left(D^{T}+D-B^{T} P B+Q_{2} S^{T} B+B^{T} S Q_{2}^{T}\right) & 0 & \left(Q_{2} S^{T}-B^{T} P\right) M \\
P A_{c} & 0 & -P & P M \\
M^{T} S Q_{1}^{T} & M^{T}\left(S Q_{2}^{T}-P B\right) & M^{T} P & -\epsilon I
\end{array}\right]<0
$$


is equivalent to $\mathcal{Z}>0, \mathcal{X}>0$, and equation (28) at the bottom of the page holds. This, by the Schur complement formula, can be shown to be equivalent to

$$
\begin{aligned}
& \mathcal{Y}_{1}+\mathcal{Y}_{2} \mathcal{X}^{-1} \mathcal{Y}_{2}^{T}+\left(\Psi_{1}^{T}+\mathcal{Y}_{2} \mathcal{X}^{-1} \Psi_{2}^{T}\right) K+K^{T}\left(\Psi_{1}+\Psi_{2} \mathcal{X}^{-1} \mathcal{Y}_{2}^{T}\right) \\
& \quad+K^{T}\left(\epsilon N_{2}^{T} N_{2}+B_{1}^{T} \mathcal{Z}^{-1} B_{1}+\Psi_{2} \mathcal{X}^{-1} \Psi_{2}^{T}\right) K<0 .
\end{aligned}
$$

It is easy to see that (29) is satisfied if and only if there exists a scalar $\delta>0$ such that $\Xi>0$ and

$$
\begin{aligned}
\mathcal{Y}_{1}+\mathcal{Y}_{2} \mathcal{X}^{-1} \mathcal{Y}_{2}^{T}+ & \left(\Psi_{1}^{T}+\mathcal{Y}_{2} \mathcal{X}^{-1} \Psi_{2}^{T}\right) K \\
& +K^{T}\left(\Psi_{1}+\Psi_{2} \mathcal{X}^{-1} \mathcal{Y}_{2}^{T}\right)+K^{T} \Xi K<0 .
\end{aligned}
$$

This can be rewritten as

$$
\begin{aligned}
\mathcal{Y}_{1} & +\mathcal{Y}_{2} \mathcal{X}^{-1} \mathcal{Y}_{2}^{T}-\left(\Psi_{1}^{T}+\mathcal{Y}_{2} \mathcal{X}^{-1} \Psi_{2}^{T}\right) \Xi^{-1}\left(\Psi_{1}+\Psi_{2} \mathcal{X}^{-1} \mathcal{Y}_{2}^{T}\right) \\
& +\left[K^{T}+\left(\Psi_{1}^{T}+\mathcal{Y}_{2} \mathcal{X}^{-1} \Psi_{2}^{T}\right) \Xi^{-1}\right] \\
& \times \Xi\left[K+\Xi^{-1}\left(\Psi_{1}+\Psi_{2} \mathcal{X}^{-1} \mathcal{Y}_{2}^{T}\right)\right]<0 .
\end{aligned}
$$

Finally, it is easy to see that there exists a matrix $K$ such that (30) holds if and only if (22) holds, and in this case, a suitable $K$ can be chosen as in (23). This completes the proof.

In the case when there is no parameter uncertainties in the uncertain discrete-time descriptor system $(\Sigma)$, this system will reduce to

$$
\begin{aligned}
(\bar{\Sigma}): \quad E x(k+1) & =A x(k)+B_{1} u(k)+B \omega(k) \\
z(k) & =C x(k)+D \omega(k)
\end{aligned}
$$

then, by Theorem 3, we have the following result.

Corollary 1: Consider the discrete-time descriptor system $(\bar{\Sigma})$. Then, there exists a state feedback controller such that the closed-loop system is admissible and ESPR if and only if there exist a scalar $\delta>0$, matrices $P>0$ and $Q$ such that

$$
\mathcal{V}=D^{T}+D-B^{T} P B+Q_{2} S^{T} B+B^{T} S Q_{2}>0
$$

and

$$
\mathcal{W}-\mathcal{U}^{T} \mathcal{T}^{-1} \mathcal{U}<0
$$

where the matrix $S \in \mathbb{R}^{n \times(n-r)}$ is given in Definition 3, and

$$
\begin{aligned}
\mathcal{W}= & Q_{1} S^{T} A+A^{T} S Q_{1}^{T}-E^{T} P E+A^{T} P A \\
& +\left[C^{T}-Q_{1} S^{T} B+A^{T}\left(S Q_{2}^{T}-P B\right)\right] \\
& \times \mathcal{V}^{-1}\left[C-B^{T} S Q_{1}^{T}+\left(Q_{2} S^{T}-B^{T} P\right) A\right] \\
\mathcal{U}= & B_{1}^{T} S Q_{1}^{T}+B_{1}^{T} P A+B_{1}^{T}\left(S Q_{2}^{T}-P B\right) \\
& \times \mathcal{V}^{-1}\left[C-B^{T} S Q_{1}^{T}+\left(Q_{2} S^{T}-B^{T} P\right) A\right] \\
\mathcal{T}= & B_{1}^{T}\left(P+\mathcal{V}^{-1}\right) B_{1}+\delta I .
\end{aligned}
$$

In this case, a suitable state feedback control law can be chosen by

$$
u(k)=-\mathcal{T}^{-1} \mathcal{U} x(k) .
$$

Remark 4: Corollary 1 provides a necessary and sufficient condition for the existence of state feedback controllers ensuring admissibility and ESPR of the closed-loop system. It is noted that the design of a desired state feedback controller involves no decomposition of the system matrices, which is due to the use of Theorem 1 where the positive definite matrix $P$ is introduced. Usually, in the synthesis of discrete-time descriptor systems, decompositions of system matrices will be involved because of the indefiniteness of the Lyapunov-type matrix [18], [20]; this will make the design procedure indirect and relatively complicated. It is also worth pointing out that in the context of discrete-time descriptor systems, the indefiniteness of the Lyapunov-type matrix may make it difficult to obtain necessary and sufficient conditions for the existence of state feedback controllers guaranteeing some performance of the closed-loop system [18], [20]. Considering the above, the results in Corollary 1 is elegant from a mathematical point of view.

\section{NUMERICAL EXAMPLE}

In this section, we give an example to demonstrate the effectiveness of the proposed method.

Consider an uncertain discrete-time descriptor system in (1) and (2) with parameters as follows:

$$
\begin{aligned}
& E=\left[\begin{array}{ccc}
1 & 3 & 0.5 \\
0 & 0 & 0 \\
0 & 0 & 0
\end{array}\right] \\
& A=\left[\begin{array}{ccc}
5.04 & 10.2 & 2.1 \\
2 & -1.5 & 1 \\
0 & 0 & 0
\end{array}\right] \\
& B_{1}=\left[\begin{array}{ccc}
-1 & -0.3 & 1 \\
0 & 1 & 0 \\
0.6 & -0.1 & 0.8
\end{array}\right] \\
& B=\left[\begin{array}{cc}
0.5 & 1 \\
1 & 0 \\
1 & -1
\end{array}\right] \\
& M=\left[\begin{array}{c}
0.1 \\
0.1 \\
-0.2
\end{array}\right] \\
& C=\left[\begin{array}{ccc}
-1.56 & 0.8 & -3.3 \\
2.3 & 2.5 & -2
\end{array}\right] \\
& D=\left[\begin{array}{cc}
5 & -1 \\
0.3 & 8.5
\end{array}\right] \\
& N_{1}=\left[\begin{array}{lll}
0.38 & 0.6 & 0.4
\end{array}\right] \\
& N_{2}=\left[\begin{array}{lll}
-0.1 & 0.1 & 0
\end{array}\right] \text {. }
\end{aligned}
$$

$$
\left[\begin{array}{cc}
\mathcal{Y}_{1}+\Psi_{1}^{T} K+K^{T} \Psi_{1}+K^{T}\left(\epsilon N_{2}^{T} N_{2}+B_{1}^{T} \mathcal{Z}^{-1} B_{1}\right) K & \mathcal{Y}_{2}+K^{T} \Psi_{2} \\
\mathcal{Y}_{2}^{T}+\Psi_{2}^{T} K & -\mathcal{X}
\end{array}\right]<0
$$


It is supposed that the uncertain matrix is given as $F(\sigma)=$ $\sin (\sigma)$. Then, it can be verified that the nominal discrete descriptor system is neither admissible nor ESPR. Now, to solve the PR control problem, we choose

$$
S=\left[\begin{array}{ll}
0 & 0 \\
1 & 0 \\
0 & 1
\end{array}\right]
$$

Then, it can be checked that

$$
\begin{aligned}
P & =\left[\begin{array}{rrr}
3 & 0.5 & 1 \\
0.5 & 2 & -0.8 \\
1 & -0.8 & 5
\end{array}\right] \\
Q_{1} & =\left[\begin{array}{rr}
-0.8 & 1.64 \\
0.5 & 1.4 \\
-2.5 & -1.7
\end{array}\right] \\
Q_{2} & =\left[\begin{array}{lr}
0.2 & 0 \\
0.1 & -0.1
\end{array}\right] \\
\delta & =0.0121 \\
\epsilon & =1.1232
\end{aligned}
$$

satisfy the matrix inequalities in (20)-(22). Therefore, by Theorem 3, we have that the PR control problem is solvable, and a desired state feedback control law can be chosen as

$$
u(k)=\left[\begin{array}{rrr}
2.5947 & 5.1464 & 1.9953 \\
-1.8499 & 1.0858 & 0.3834 \\
-2.8844 & -4.3098 & -0.7825
\end{array}\right] x(k)
$$

\section{CONCLUSION}

The problems of PR analysis and PR control for discrete-time descriptor systems with parameter uncertainties in both the state and input matrices have been studied. In terms of a strict LMI, a new necessary and sufficient condition for a discrete-time descriptor system to be regular, causal, stable and ESPR have been proposed. The problems are solved via the notions of strong robust admissibility with ESPR and strong robust admissibilizability with ESPR. Without any additional assumptions on the system matrices, necessary and sufficient conditions for strong robust admissibility with ESPR and strong robust admissibilizability with ESPR have been obtained in terms of a strict LMI and matrix inequalities, respectively. An explicit construction procedure to obtain a desired state feedback control law has also been given, which involves no decomposition of the system matrices.

\section{APPENDIX \\ PROOF OF THEOREM 1}

\section{A. Sufficiency}

Assume that the LMI in (11) is satisfied. We first show the admissibility of the system $(\hat{\Sigma})$. To this end, we write

$$
Q=\left[\begin{array}{l}
Q_{1} \\
Q_{2}
\end{array}\right]
$$

where $Q_{1} \in \mathbb{R}^{n \times(n-r)}$ and $Q_{2} \in \mathbb{R}^{l \times(n-r)}$. Then, the LMI in (11) can be rewritten as (33) shown at the bottom of the page, which implies

$$
A^{T} P A-E^{T} P E+A^{T} S Q_{1}^{T}+Q_{1} S^{T} A<0 .
$$

Therefore, by Lemma 1 , we have that system $(\hat{\Sigma})$ is admissible. Next, we show that under the condition of the theorem, the discrete-time descriptor system $(\hat{\Sigma})$ is ESPR. By the Schur complement formula, it follows from (33) that

$$
A^{T} P A-E^{T} P E+A^{T} S Q_{1}^{T}+Q_{1} S^{T} A+\Omega<0
$$

where

$$
\begin{aligned}
\Omega= & \left(C^{T}-A^{T} P B+A^{T} S Q_{2}^{T}-Q_{1} S^{T} B\right) \\
& \times U^{-1}\left(C-B^{T} P A+Q_{2} S^{T} A-B^{T} S Q_{1}^{T}\right) \\
U= & D^{T}+D-B^{T} P B+B^{T} S Q_{2}^{T}+Q_{2} S^{T} B>0
\end{aligned}
$$

Then, it is easy to see that (34) implies that there exists a matrix $W>0$ such that

$$
A^{T} P A-E^{T} P E+A^{T} S Q_{1}^{T}+Q_{1} S^{T} A+\Omega+W<0 .
$$

Let

$$
\Phi(j \theta)=e^{j \theta} E-A
$$

Then, recalling that the system $(\hat{\Sigma})$ is admissible, we have that $\Phi(j \theta)$ is nonsingular for all $\theta \in[0,2 \pi)$. Pre- and post-multiplying (37) by $B^{T} \Phi(-j \theta)^{-T}$ and $\Phi(j \theta)^{-1} B$, respectively, yield

$$
\begin{aligned}
& B^{T} \Phi(-j \theta)^{-T}\left[A^{T} P A-E^{T} P E+A^{T} S Q_{1}^{T}+Q_{1} S^{T} A\right] \\
& \times \Phi(j \theta)^{-1} B+B^{T} \Phi(-j \theta)^{-T}(\Omega+W) \Phi(j \theta)^{-1} B \leq 0
\end{aligned}
$$

which implies

$$
\begin{gathered}
-B^{T} \Phi(-j \theta)^{-T}\left[A^{T} P A-E^{T} P E+A^{T} S Q_{1}^{T}+Q_{1} S^{T} A\right] \\
\quad \times \Phi(j \theta)^{-1} B \geq B^{T} \Phi(-j \theta)^{-T}(\Omega+W) \Phi(j \theta)^{-1} B .
\end{gathered}
$$

$$
\left[\begin{array}{cc}
A^{T} P A-E^{T} P E+A^{T} S Q_{1}^{T}+Q_{1} S^{T} A & C^{T}-A^{T} P B+A^{T} S Q_{2}^{T}-Q_{1} S^{T} B \\
C-B^{T} P A+Q_{2} S^{T} A-B^{T} S Q_{1}^{T} & -\left(D^{T}+D-B^{T} P B+B^{T} S Q_{2}^{T}+Q_{2} S^{T} B\right)
\end{array}\right]<0
$$


Now, by some simple algebraic manipulations, it can be verified that

$$
\begin{aligned}
B^{T} P B & +B^{T} \Phi(-j \theta)^{-T}\left(A^{T} P+Q_{1} S^{T}\right) B \\
& +B^{T}\left(A^{T} P+Q_{1} S^{T}\right)^{T} \Phi(j \theta)^{-1} B \\
= & -B^{T} \Phi(-j \theta)^{-T}\left(A^{T} P A-E^{T} P E+A^{T} S Q_{1}^{T}\right. \\
& \left.+Q_{1} S^{T} A\right) \Phi(j \theta)^{-1} B
\end{aligned}
$$

where the relationship $E^{T} S=0$ is used. From (38) and (40), it is easy to show that for all $\theta \in[0,2 \pi)$

$$
\begin{aligned}
B^{T} P B & +B^{T} \Phi(-j \theta)^{-T}\left(A^{T} P+Q_{1} S^{T}\right) B \\
& +B^{T}\left(A^{T} P+Q_{1} S^{T}\right)^{T} \Phi(j \theta)^{-1} B \\
\geq & B^{T} \Phi(-j \theta)^{-T}(\Omega+W) \Phi(j \theta)^{-1} B .
\end{aligned}
$$

Note that for all $\theta \in[0,2 \pi)$

$$
\begin{aligned}
& G\left(e^{j \theta}\right)+G\left(e^{j \theta}\right)^{*} \\
&=D+D^{T}+C \Phi(j \theta)^{-1} B+\left(C \Phi(j \theta)^{-1} B\right)^{*} \\
&=U+B^{T} P B+\left(C-B^{T} P A+Q_{2} S^{T} A-B^{T} S Q_{1}^{T}\right) \Phi(j \theta)^{-1} B \\
&+B^{T} \Phi(-j \theta)^{-T}\left(C^{T}-A^{T} P B+A^{T} S Q_{2}^{T}-Q_{1} S^{T} B\right) \\
&+\left[\left(B^{T} P A-Q_{2} S^{T} A+B^{T} S Q_{1}^{T}\right) \Phi(j \theta)^{-1}-Q_{2} S^{T}\right] B \\
&+B^{T}\left[\Phi(-j \theta)^{-T}\left(A^{T} P B-A^{T} S Q_{2}^{T}+Q_{1} S^{T} B\right)-S Q_{2}^{T}\right] \\
&= U+\left(C-B^{T} P A+Q_{2} S^{T} A-B^{T} S Q_{1}^{T}\right) \Phi(j \theta)^{-1} B \\
&+B^{T} \Phi(-j \theta)^{-T}\left(C^{T}-A^{T} P B+A^{T} S Q_{2}^{T}-Q_{1} S^{T} B\right) \\
&+B^{T} P B+B^{T} \Phi(-j \theta)^{-T}\left(A^{T} P+Q_{1} S^{T}\right) B+B^{T}\left(A^{T} P\right. \\
&\left.+Q_{1} S^{T}\right)^{T} \Phi(j \theta)^{-1} B
\end{aligned}
$$

where $U$ is given in (35). Then, by (41) and (42), we have that for all $\theta \in[0,2 \pi)$

$$
\begin{aligned}
& G\left(e^{j \theta}\right)+G\left(e^{j \theta}\right)^{*} \\
& \geq U+\left(C-B^{T} P A+Q_{2} S^{T} A-B^{T} S Q_{1}^{T}\right) \Phi(j \theta)^{-1} B \\
& \quad+B^{T} \Phi(-j \theta)^{-T}\left(C^{T}-A^{T} P B+A^{T} S Q_{2}^{T}-Q_{1} S^{T} B\right) \\
& \quad+B^{T} \Phi(-j \theta)^{-T}(\Omega+W) \Phi(j \theta)^{-1} B .
\end{aligned}
$$

Noting $\Omega+W>0$ and using Lemma 2, we obtain

$$
\begin{aligned}
& G\left(e^{j \theta}\right)+G\left(e^{j \theta}\right)^{*} \geq U-\left(C-B^{T} P A+Q_{2} S^{T} A-B^{T} S Q_{1}^{T}\right) \\
& \quad \times(\Omega+W)^{-1}\left(C^{T}-A^{T} P B+A^{T} S Q_{2}^{T}-Q_{1} S^{T} B\right) .
\end{aligned}
$$

Observe that

$$
\begin{aligned}
\Omega+W- & \left(C^{T}-A^{T} P B+A^{T} S Q_{2}^{T}-Q_{1} S^{T} B\right) U^{-1} \\
& \times\left(C-B^{T} P A+Q_{2} S^{T} A-B^{T} S Q_{1}^{T}\right)=W>0
\end{aligned}
$$

which, by the Schur complement formula, implies (44) shown at the bottom of the page. By the Schur complement formula again, it follows from (44) that

$$
\begin{array}{r}
U-\left(C-B^{T} P A+Q_{2} S^{T} A-B^{T} S Q_{1}^{T}\right)(\Omega+W)^{-1} \\
\times\left(C^{T}-A^{T} P B+A^{T} S Q_{2}^{T}-Q_{1} S^{T} B\right)>0
\end{array}
$$

Therefore, from (43) and (45), we have that for all $\theta \in[0,2 \pi)$

$$
G\left(e^{j \theta}\right)+G^{*}\left(e^{j \theta}\right)>0 .
$$

On the other hand, since $(\hat{\Sigma})$ is admissible, we can find two nonsingular matrices $\tilde{M}_{1}$ and $\tilde{N}_{1}$ such that [2]

$$
\tilde{M}_{1} E \tilde{N}_{1}=\left[\begin{array}{ll}
I & 0 \\
0 & 0
\end{array}\right] \quad \tilde{M}_{1} A \tilde{N}_{1}=\left[\begin{array}{cc}
\tilde{A}_{1} & 0 \\
0 & I
\end{array}\right]
$$

In this case, the matrix $S$ satisfying $E^{T} S=0$ can be chosen as

$$
S=\tilde{M}_{1}^{T}\left[\begin{array}{l}
0 \\
I
\end{array}\right] \tilde{H}_{1}
$$

where $\tilde{H}_{1}$ is any nonsingular matrix. Now, write

$$
\begin{aligned}
C \tilde{N}_{1} & =\left[\begin{array}{ll}
\tilde{C}_{1} & \tilde{C}_{2}
\end{array}\right] \\
\tilde{M}_{1} B & =\left[\begin{array}{l}
\tilde{B}_{1} \\
\tilde{B}_{2}
\end{array}\right] \\
\tilde{M}_{1}^{-T} P \tilde{M}_{1}^{-1} & =\left[\begin{array}{ll}
\tilde{P}_{1} & \tilde{P}_{2} \\
\tilde{P}_{2}^{T} & \tilde{P}_{3}
\end{array}\right] \\
\tilde{N}_{1}^{T} Q_{1} \tilde{H}_{1}^{T} & =\left[\begin{array}{l}
\tilde{Q}_{11} \\
\tilde{Q}_{21}
\end{array}\right] \\
Q_{2} \tilde{H}_{1}^{T} & =\tilde{Q}_{2}
\end{aligned}
$$

where the partition is compatible with that of $\tilde{M}_{1} E \tilde{N}_{1}$ and $\tilde{M}_{1} A \tilde{N}_{1}$ in (47). Then, it is easy to see that

$$
\tilde{P}_{1}>0
$$

Pre- and post-multiplying (33) by $\operatorname{diag}\left(\tilde{N}_{1}^{T}, I\right)$ and $\operatorname{diag}\left(\tilde{N}_{1}, I\right)$, respectively and then using the above notations, we have (49) shown at the bottom of the page. Where

$$
\left[\begin{array}{cc}
U & C-B^{T} P A+Q_{2} S^{T} A-B^{T} S Q_{1}^{T} \\
C^{T}-A^{T} P B+A^{T} S Q_{2}^{T}-Q_{1} S^{T} B & \Omega+W
\end{array}\right]>0
$$

$$
\left[\begin{array}{ccc}
* & \tilde{P}_{3}^{*}+\tilde{Q}_{21}+\tilde{Q}_{21}^{T} & \tilde{C}_{2}^{T}-\tilde{P}_{2}^{T} \tilde{B}_{1}-\tilde{P}_{3}^{*} \tilde{B}_{2}+\tilde{Q}_{2}^{T}-\tilde{Q}_{21} \tilde{B}_{2} \\
* & \tilde{C}_{2}^{T} & \mathcal{V}
\end{array}\right]<0
$$


* represents matrices that will not be used in the following discussion, and

$$
\begin{aligned}
\mathcal{V}=-D^{T}-D+ & \tilde{B}_{1}^{T} \tilde{P}_{1} \tilde{B}_{1}+\tilde{B}_{2}^{T} \tilde{P}_{2}^{T} \tilde{B}_{1} \\
& +\tilde{B}_{1}^{T} \tilde{P}_{2} \tilde{B}_{2}+\tilde{B}_{2}^{T} \tilde{P}_{3} \tilde{B}_{2}-\tilde{B}_{2}^{T} \tilde{Q}_{2}^{T}-\tilde{Q}_{2} \tilde{B}_{2} .
\end{aligned}
$$

Pre- and post-multiplying (49) by

$$
\left[\begin{array}{ccc}
I & 0 & 0 \\
0 & I & 0 \\
0 & \tilde{B}_{2}^{T} & I
\end{array}\right]
$$

and its transpose, respectively, and then noting the 3-3 block, we obtain

$$
-D^{T}-D+\tilde{C}_{2} \tilde{B}_{2}+\tilde{B}_{2}^{T} \tilde{C}_{2}^{T}+\tilde{B}_{1}^{T} \tilde{P}_{1} \tilde{B}_{1}<0
$$

This together with (48) gives

$$
D-\tilde{C}_{2} \tilde{B}_{2}+D^{T}+\tilde{B}_{2}^{T} \tilde{C}_{2}^{T}>0
$$

which together with

$$
G(z)=C(z E-A)^{-1} B=\tilde{C}_{1}\left(z I-\tilde{A}_{1}\right)^{-1} \tilde{B}_{1}-\tilde{C}_{2} \tilde{B}_{2}+D
$$

implies

$$
G(\infty)+G(\infty)^{T}>0
$$

Considering this and (46), it easy to see that the discrete-time descriptor system $(\hat{\Sigma})$ is ESPR.

\section{B. Necessity}

Suppose that the discrete-time descriptor system $(\hat{\Sigma})$ is admissible and ESPR. Then, there exist two nonsingular matrices $\mathcal{M}$ and $\mathcal{N}$ such that [2]

$$
E=\mathcal{M}\left[\begin{array}{ll}
I & 0 \\
0 & 0
\end{array}\right] \mathcal{N} \quad A=\mathcal{M}\left[\begin{array}{cc}
\mathcal{A} & 0 \\
0 & I
\end{array}\right] \mathcal{N}
$$

Then, it is easy to see that the matrix $S$ satisfying $E^{T} S=0$ can be written as

$$
S=\mathcal{M}^{-T}\left[\begin{array}{l}
0 \\
I
\end{array}\right] \mathcal{H}
$$

where $\mathcal{H}$ is any nonsingular matrix. Write

$$
B=\mathcal{M}\left[\begin{array}{l}
B_{1} \\
B_{2}
\end{array}\right] \quad C=\left[\begin{array}{ll}
C_{1} & C_{2}
\end{array}\right] \mathcal{N}
$$

where the partition is compatible with that of $A$. Note that

$$
G(z)=C_{1}(z I-\mathcal{A})^{-1} B_{1}+D-C_{2} B_{2} .
$$

Considering the system $(\hat{\Sigma})$ is ESPR, by Lemma 3 , it follows that there exists a matrix $\tilde{P}>0$ such that

$$
\left[\begin{array}{cc}
\mathcal{A}^{T} \tilde{P} \mathcal{A}-\tilde{P} & C_{1}^{T}-\mathcal{A}^{T} \tilde{P} B_{1} \\
C_{1}-B_{1}^{T} \tilde{P} \mathcal{A} & -\left(D^{T}+D-C_{2} B_{2}-B_{2}^{T} C_{2}^{T}-B_{1}^{T} \tilde{P} B_{1}\right)
\end{array}\right]<0 .
$$

Let

$$
Q_{1}=0 \quad Q_{2}=-I \quad Q_{3}=-C_{2}+B_{2}^{T}
$$

Then, by (52), it is easy to see that we obtain the first equation of the bottom of the page. Pre- and post-multiplying this inequality by

$$
\left[\begin{array}{ccc}
I & 0 & 0 \\
0 & 0 & I \\
0 & I & -B_{2}^{T}
\end{array}\right]
$$

and its transpose, respectively, we obtain (53) shown at the bottom of the page. Set

$$
\mathcal{P}=\mathcal{M}^{-T}\left[\begin{array}{ll}
\tilde{P} & 0 \\
0 & I
\end{array}\right] \mathcal{M} \quad \mathcal{Q}=\left[\begin{array}{l|l}
\mathcal{N} & 0 \\
\hline 0 & I
\end{array}\right]^{T}\left[\begin{array}{l}
Q_{1} \\
Q_{2} \\
\hline Q_{3}
\end{array}\right] \mathcal{H}^{-T}
$$

Then, pre- and post-multiplying (53) by

$$
\left[\begin{array}{l|l}
\mathcal{N} & 0 \\
\hline 0 & I
\end{array}\right]^{T}
$$

and its transpose, we can write

$$
\begin{aligned}
& {\left[\begin{array}{cc}
A^{T} \mathcal{P} A-E^{T} \mathcal{P} E & C^{T}-A^{T} \mathcal{P} B \\
C-B^{T} \mathcal{P} A & -\left(D^{T}+D-B^{T} \mathcal{P} B\right)
\end{array}\right]} \\
& +\left[\begin{array}{c}
A^{T} \\
-B^{T}
\end{array}\right] S \mathcal{Q}^{T}+\mathcal{Q} S^{T}\left[\begin{array}{c}
A^{T} \\
-B^{T}
\end{array}\right]^{T}<0 .
\end{aligned}
$$

That is, the matrices $\mathcal{P}$ and $\mathcal{Q}$ given in (54) satisfy (55). This completes the proof.

$$
\left[\begin{array}{ccc}
\mathcal{A}^{T} \tilde{P} \mathcal{A}-\tilde{P} & C_{1}^{T}-\mathcal{A}^{T} \tilde{P} B_{1} & Q_{1} \\
C_{1}-B_{1}^{T} \tilde{P} \mathcal{A} & -\left(D^{T}+D-C_{2} B_{2}-B_{2}^{T} C_{2}^{T}-B_{1}^{T} \tilde{P} B_{1}\right) & C_{2}+Q_{3}+B_{2}^{T} Q_{2} \\
Q_{1}^{T} & C_{2}^{T}+Q_{3}^{T}+Q_{2}^{T} B_{2} & I+Q_{2}+Q_{2}^{T}
\end{array}\right]<0
$$

$$
\left[\begin{array}{cc|c}
\mathcal{A}^{T} \tilde{P} \mathcal{A}-\tilde{P} & Q_{1} & C_{1}^{T}-\mathcal{A}^{T} \tilde{P} B_{1}-Q_{1} B_{2} \\
Q_{1}^{T} & I+Q_{2}+Q_{2}^{T} & C_{2}^{T}-B_{2}+Q_{3}^{T}-Q_{2} B_{2} \\
\hline C_{1}-B_{1}^{T} \tilde{P} \mathcal{A}-B_{2}^{T} Q_{1}^{T} & C_{2}-B_{2}^{T}+Q_{3}-B_{2}^{T} Q_{2}^{T} & -D^{T}-D+B_{1}^{T} \tilde{P} B_{1}+B_{2}^{T} B_{2}-B_{2}^{T} Q_{3}^{T}-Q_{3} B_{2}
\end{array}\right]<0
$$




\section{REFERENCES}

[1] J. Bernussou, J. C. Geromel, and M. C. De Oliveira, "On strict positive real systems design: Guaranteed cost and robustness issues," Syst. Contr. Lett., vol. 36, pp. 135-141, 1999.

[2] L. Dai, Singular Control Systems. Berlin, Germany: Springer-Verlag, 1989.

[3] W. M. Haddad and D. S. Bernstein, "Robust stabilization with positive real uncertainty: Beyond the small gain theorem," Syst. Control Lett., vol. 17, pp. 191-208, 1991

[4] — - "Explicit construction of quadratic Lyapunov functions for the small gain, positivity, circle, and Popov theorems and their application to robust stability. Part II: Discrete-time theory," Int. J. Robust Nonlinear Control, vol. 4, pp. 249-265, 1994.

[5] F. L. Lewis, "A survey of linear singular systems," Circuits, Syst. Signal Processing, vol. 5, pp. 3-36, 1986.

[6] C. Lin, J. Wang, D. Wang, and C. B. Soh, "Robustness of uncertain descriptor systems," Syst. Control Lett., vol. 31, pp. 129-138, 1997.

[7] M. S. Mahmoud, Y. C. Soh, and L. Xie, "Observer-based positive real control of uncertain linear systems," Automatica, vol. 35, pp. 749-754, 1999.

[8] M. S. Mahmoud and L. Xie, "Positive real analysis and synthesis of uncertain discrete time systems," IEEE Trans. Circuits Syst. I, vol. 47, pp. 403-406, Mar. 2000.

[9] I. R. Petersen, "A stabilization algorithm for a class of uncertain linear systems," Syst. Control Lett., vol. 8, pp. 351-357, 1987.

[10] W. Sun, P. P. Khargonekar, and D. Shim, "Solution to the positive real control problem for linear time-invariant systems," IEEE Trans. Automat. Contr., vol. 39, pp. 2034-2046, Oct. 1994.

[11] E. Uezato and M. Ikeda, "Strict LMI conditions for stability, robust stabilization, and $H_{\infty}$ control of descriptor systems," in Proc. 38th IEEE Conf. Decision and Control, Phoenix, AZ, Dec. 1999, pp. 4092-4097.

[12] M. Vidyasagar, Nonlinear Systems Analysis. Englewood Cliffs, NJ: Prentice-Hall, 1993

[13] L. Xie and C. E. de Souza, "Robust $H_{\infty}$ control for linear time-invariant systems with norm-bounded uncertainty in the input matrix," Syst. Control Lett., vol. 14, pp. 389-396, 1990.

[14] L. Xie and Y. C. Soh, "Positive real control for uncertain linear timeinvariant systems," Syst. Control Lett., vol. 24, pp. 265-271, 1995.

[15] S. Xu and J. Lam, "Robust stability and stabilization of discrete singular systems: An equivalent characterization," IEEE Trans. Automat. Contr., vol. 49, pp. 568-574, Apr. 2004

[16] S. Xu, J. Lam, Z. Lin, and K. Galkowski, "Positive real control for uncertain two-dimensional systems," IEEE Trans. Circuits Syst. I, vol. 49, pp. 1659-1666, Nov. 2002.

[17] S. Xu, J. Lam, C. Yang, and E. I. Verriest, "An LMI approach to guaranteed cost control for uncertain linear neutral delay systems," Int. J. Robust Nonlinear Control, vol. 13, pp. 35-53, 2003.

[18] S. Xu and C. Yang, "Stabilization of discrete-time singular systems: A matrix inequalities approach," Automatica, vol. 35, pp. 1613-1617, 1999.

[19] — "An algebraic approach to the robust stability analysis and robust stabilization of uncertain singular systems," Int. J. Syst. Sci., vol. 31, pp. $55-61,2000$.

[20] S. Xu, C. Yang, Y. Niu, and J. Lam, "Robust stabilization for uncertain discrete singular systems," Automatica, vol. 37, pp. 769-774, 2001.

[21] L. Zhang, J. Lam, and S. Xu, "On positive realness of descriptor systems," IEEE Trans. Circuits Syst. I, vol. 49, pp. 401-407, Mar. 2002

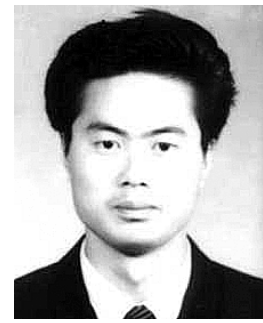

Shengyuan $\mathrm{Xu}$ received the B.Sc. degree from Hangzhou Normal University, Hangzhou, China, the M.Sc. degree from Qufu Normal University, Qufu, China, and the Ph.D. degree from Nanjing University of Science and Technology, Nanjing, China, in 1990, 1996, and 1999, respectively.

From 1999 to 2000, he was a Research Associate in the Department of Mechanical Engineering, University of Hong Kong, Hong Kong. From December 2000 to November 2001, and December 2001 to September 2002, he was a Postdoctoral Researcher at CESAME, Universitè catholique de Louvain, Louvain-la-Neuve, Belgium, and the Department of Electrical and Computer Engineering, University of Alberta, Edmonton, Canada, respectively. From September 2002 to August 2003, he was a William Mong Young Researcher in the Department of Mechanical Engineering, University of Hong Kong. He is a Professor and Ph.D. Supervisor in the Department of Automation, Nanjing University of Science and Technology, and an Honorary Associate Professor in the Department of Mechanical Engineering, University of Hong Kong. His current research interests include robust filtering and control, singular systems, time-delay systems, multidimensional systems and nonlinear systems. He has published more than 40 papers in international scientific journals.

Dr. Xu was a recipient of the National Excellent Doctoral Dissertation Award in the year 2002 from the National Education Commission of China.

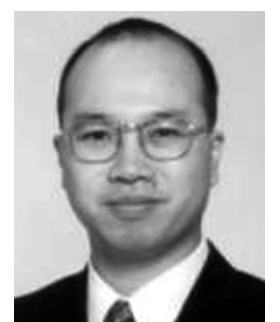

James Lam (S'86-M'88-SM'99) received the B.Sc. degree (first class) in mechanical engineering from the University of Manchester, Manchester, U.K., in 1983, and the M.Phil. and Ph.D. degrees in control engineering from the University of Cambridge, Cambridge, U.K., in 1985 and 1988, respectively.

His postdoctoral research was carried out in the Australian National University, Canberra, Australia, between 1990 and 1992.

Dr. Lam has held faculty positions at the City University of Hong Kong, Hong Kong, and the University of Melbourne, Melbourne, Australis. He is an Associate Professor in the Department of Mechanical Engineering, the University of Hong Kong, and holds a Concurrent Professorship at the Northeastern University, China, a Guest Professorhsip at the Huazhong University of Science and Technology, China, a Consulting Professorship at the South China University of Technology, China, and a Guest Professorship at Shandong University, China. His research interests include model reduction, delay systems, descriptor systems, stochastic systems, multidimensional systems, robust control and filtering, fault detection, and reliable control. He is an Associate Editor of the Asian Journal of Control, and the Interational Journal of Applied Mathematics and Computer Science.

Dr. Lam was awarded the Ashbury Scholarship, the A.H. Gibson Prize and the H. Wright Baker Prize for his academic performance at the University of Manchester. He is a Chartered Mathematician, a Fellow of the Institute of Mathematics and Its Applications (U.K.), a Member of the Institution of Electrical Engineers (U.K.), and is a Scholar (1984) and Fellow (1990) of the Croucher Foundation. 